\title{
Ankrd45 Is a Novel Ankyrin Repeat Protein Required for Cell Proliferation
}

\author{
Yunsi Kang ${ }^{1, \dagger}$, Haibo Xie ${ }^{1,+}$ and Chengtian Zhao $1,2,3, * \mathbb{D}$ \\ 1 Institute of Evolution \& Marine Biodiversity, Ocean University of China, Qingdao 266003, China; \\ kangyunsi@stu.ouc.edu.cn (Y.K.); xiehaibo@stu.ouc.edu.cn (H.X.) \\ 2 Laboratory for Marine Biology and Biotechnology, Qingdao National Laboratory for Marine Science and \\ Technology, Qingdao 266003, China \\ 3 Ministry of Education Key Laboratory of Marine Genetics and Breeding, College of Marine Life Sciences, \\ Ocean University of China, Qingdao 266003, China \\ * Correspondence: chengtian_zhao@ouc.edu.cn \\ + These authors contributed equally to this work.
}

Received: 27 May 2019; Accepted: 11 June 2019; Published: 16 June 2019

\begin{abstract}
Ankyrin repeats, the most common protein-protein interaction motifs in nature, are widely present in proteins of both eukaryotic and prokaryotic cells. Ankyrin repeat-containing proteins play diverse biological functions. Here, we identified the gene ankrd45, which encodes a novel, two ankyrin repeat-containing protein. Zebrafish ankrd45 displayed a tissue specific expression pattern during early development, with high expression in ciliated tissues, including otic vesicles, Kupffer's vesicles, pronephric ducts, and floor plates. Surprisingly, zebrafish ankrd45 mutants were viable and developed grossly normal cilia. In contrast, mutant larvae developed enlarged livers when induced with liver specific expression of Kras ${ }^{\mathrm{G} 12 \mathrm{~V}}$, one of the common mutations of KRAS that leads to cancer in humans. Further, histological analysis suggested that multiple cysts developed in the mutant liver due to cell apoptosis. Similarly, knockdown of ANKRD45 expression with either siRNA or CRISPR/Cas9 methods induced apoptosis in cultured cells, similar to those in zebrafish ankrd45 mutant livers after induction. Using different cell lines, we show that the distribution of ANKRD45 protein was highly dynamic during mitosis. ANKRD45 is preferably localized to the midbody ring during cytokinesis. Together, our results suggest that ANKRD45 is a novel ankyrin repeat protein with a conserved role during cell proliferation in both zebrafish embryos and mammalian cells.
\end{abstract}

Keywords: ANKRD45; zebrafish; KRAS; cell cycle; apoptosis; cancer

\section{Introduction}

The ankyrin repeat (ANK), a motif of 33 amino acid residues, is one of the most common protein motifs that is widely present in all forms of life, including bacteria, archaea, eukaryotes, and viruses. ANK proteins often contain such motifs in tandem arrays with each exhibiting a canonical helix-turn-helix conformation, a well-known motif involved in protein-protein interactions [1,2]. ANK repeats were first identified in the yeast cell cycle regulator Swi6/Cdc10 and the Drosophila cell signaling Notch protein, and later named after the human membrane-associated ankyrin protein, which contains 24 such repeats and regulates the interaction between the cytoskeleton and the plasma membrane $[3,4]$.

Currently, thousands of ANK-containing proteins have been identified, which perform a wide range of functions, including signal transduction, cell cycle regulation, vesicle trafficking, cytoskeletal organization, and transcriptional regulation [2,5]. Dysfunction of ANK proteins is associated with many human disorders. Mutation of $\mathrm{p} 16$, a tumor suppressor protein with four ankyrin repeats, 
is associated with several human cancers due to abnormal cell cycle defects [6,7]. Disruption in the ankyrin repeat domains in Notch proteins leads to neurological disorders in humans $[8,9]$. The ANK protein $\mathrm{I} \kappa \mathrm{B}$, an inhibitor of nuclear factor kappa B (NF- $\mathrm{kB}$ ), is involved in transcription regulation and mediates metabolism and inflammatory responses [10,11]. IKB may also induce apoptosis in cancer cells as inhibition of IKKa, an IKB kinase leading to its degradation, can switch the effects of estrogens on human breast cancer MCF-7 cells from anti- to pro-apoptotic [12,13]. Inversin (INVS), also known as NPHP2, is a ciliary-localizing protein with multiple ANK domains. Patients harboring mutations in the INVS gene manifest multiple defects, including renal cystic disease and left-right asymmetry defects due to abnormal functioning of cilia [14].

Cilia are tiny organelles protruding from the cell surface and perform diverse biological functions [15]. Dysfunction of cilia may lead to multiple defects during embryonic development and result in a class of genetic disorders collectively termed as ciliopathies [16]. Recently, zebrafish have been used as disease models for ciliopathies [17]. Cilia are present in various organs of developing zebrafish larvae. Particularly, the olfactory pits, pronephric ducts, floor plates, and Kupffer's vesicles are tissues rich in motile cilia, and cilia genes are often expressed at a higher level in these organs [17]. Zebrafish cilia mutants frequently develop curly body axis phenotype due to motile cilia defects in the spinal cord [18].

KRAS, together with HRAS and NRAS, are members of the RAS family of small GTPases and mutations of these RAS genes are associated with one third of human cancers [19]. The KRAS ${ }^{G 12 V}$ mutation is one of the common mutations that is found in many human cancers $[19,20]$. The G12V oncogenic mutation renders the KRAS protein more active by diminishing its hydrolysis from the GTP-bound active state to the GDP-bound inactive state. The GTP-bound KRASG12V proteins chronically bind to and activate multiple downstream signaling pathways, including MAPK or PI3K/AKT signals, which lead to excessive cell proliferation and subsequent carcinogenesis [20].

In this study, we report the functions of a novel ANK protein, ANKRD45. We show that ankrd45 exhibits a tissue specific expression pattern with high enrichment in ciliary tissues during early zebrafish development. Although zebrafish ankrd45 mutants were viable with grossly normal cilia, mutant larvae displayed proliferation defects when induced with a liver specific $\mathrm{kras}^{\mathrm{G} 12 \mathrm{~V}}$ transgene. We further investigated the role of ANKRD45 both in zebrafish and in cell lines. Our data suggests that ANKRD45 is a novel player during cell cycle regulation.

\section{Materials and Methods}

\subsection{Zebrafish Strains}

All zebrafish strains were maintained at a $14 \mathrm{~h}$ light/10 h dark cycle at $28.5^{\circ} \mathrm{C}$. The Tet-on inducible double transgene Tg (fabp10:rtTA2s-M2;TRE2:EGFP-kras ${ }^{G 12 V}$ ) (Gift from Dr. Gong, NUS) was used to generate the liver tumor model [21,22]. The ankrd45 mutants were generated using the CRISPR/Cas9 system with the following target sequencing for sgRNA synthesis: 5'-GGTGTCCAGCTGACCCCACA-3'.

\subsection{Whole Mount In Situ Hybridization and Immunohistochemistry}

Full-length ankrd45 gene was amplified from 24-h post fertilization (hpf) zebrafish cDNA with the following primers: Forward 5'-CACACCACATCACTACTCTTC-3', Reverse 5'-GTAATGCAGTCCAACAGTTTC-3'. The PCR products were ligated into pEASY-T3 vectors. Probe preparation and hybridization were performed using standard protocols. To analyze its expression in liver, zebrafish larvae were anaesthetized and fixed at 5 days post fertilization (dpf) for cryosectioning. Transverse sections through the liver were collected for fluorescence in situ hybridization analysis. Fluorescent signal amplification using a TSA-plus Fluorescein System (Perkin Elmer Life Sciences, Boston, MA, USA) was carried out according to the manufacturer's protocols. For immunohistochemistry, the anti-monoglycylated Tubulin Antibody (TAP952, Merck-Millipore, 
Darmstadt, Germany) was used to visualize cilia in wild-type and mutant larvae. The antibody staining procedure was performed as described previously [23].

\subsection{Pharmaceutical Treatments and $m R N A$ Overexpression}

To evaluate liver development, we generated wild-type and ankrd45 homozygous mutants carrying the $T g$ (fabp10:rtTA2s-M2;TRE2:EGFP-kras ${ }^{G 12 V}$ ) transgene. In our experience, we have found that the homozygous EGFP-kras ${ }^{G 12 V}$ transgene developed larger livers compared with the heterozygote transgene in wild-type larvae. To exclude this situation, we crossed ankrd45 homozygous mutants carrying the EGFP-kras ${ }^{G 12 V}$ transgene with ankrd45 homozygous mutants, and compared them with wild-type embryos carrying the heterozygote transgene. The collected embryos were treated with $60 \mu \mathrm{g} / \mathrm{mL}$ doxycycline hydrochloride (Sangon Biotech, Shanghai, China) or DMSO starting from $60 \mathrm{hpf}$. Images of the liver with green fluorescence were captured using a Leica M165FC microscope at different time points after treatment. The size of liver was evaluated using Image J software according to the area of green fluorescent protein (GFP) expression.

For rescue experiments, full length ankrd45 cDNA was subcloned into pCS2+ expression vectors. After linearization, mRNA was transcribed using the mMessage mMachine Sp6 transcription kit (Invitrogen-Ambion, Austin, TX, USA). Microinjections were performed at the one-cell stage at a concentration of $100 \mathrm{ng} / \mu \mathrm{L}$. Injected embryos were maintained for drug treatments at later stages to evaluate liver development.

\subsection{Histological Analysis and TUNEL Assay}

Zebrafish larvae carrying the EGFP-kras ${ }^{G 12 V}$ transgene were fixed at three days after doxycycline treatment in $4 \%$ paraformaldehyde (PFA) $(w / v)$ in PBST overnight at $4{ }^{\circ} \mathrm{C}$. After gradually dehydrating in ethanol, the fixed embryos were finally embedded in JB4 embedding medium (Polysciences Inc., Warrington, PA, USA). Transverse sections through the liver were collected using Leica RM2235 microtome and stained with hematoxylin and eosin. Images were taken using a Leica DM2500 microscope. For TUNEL analysis, the doxycycline-treated embryos were fixed in $4 \%$ PFA overnight at $4{ }^{\circ} \mathrm{C}$, infiltrated in $30 \%$ sucrose overnight at $4{ }^{\circ} \mathrm{C}$, then embedded in frozen medium for cryosectioning using LEICA cryostat (CM1860). The sections were collected using adhesive slides and air dried for one hour at room temperature (RT). After that, sections were incubated in $70 \%$ ethanol $/ 30 \%$ glacial acetic acid at $-20^{\circ} \mathrm{C}$ for $5 \mathrm{~min}$, washed 5 times with $50 \mathrm{mM}$ Tris- $\mathrm{HCl}$, then blocked for $30 \mathrm{~min}$ at RT with blocking solution ( $1 \times \mathrm{PBS} / 10 \%$ normal goat serum/0.5\% TrionX-100). The TUNEL assay was performed using the in situ cell death detection kit (Roche, Indianapolis, IN, USA) according to standard protocols from the manufacturer.

\subsection{BrdU Incorporation Assay}

At $68 \mathrm{~h}$ after doxycycline inducement, zebrafish larvae were incubated in $10 \mathrm{mM}$ BrdU (Sigma, St. Louis, MO, USA) in $1 \%$ DMSO for four hours at $28.5^{\circ} \mathrm{C}$, then anesthetized in tricaine and fixed with $4 \%$ paraformaldehyde for two hours at RT. The fixed embryos were then sent for cryosectioning. Sections were treated with $2 \mathrm{~N} \mathrm{HCl}$ for $1 \mathrm{~h}$ at $\mathrm{RT}$, blocked $1 \mathrm{~h}$ with blocking solution, then incubated with mouse anti-BrdU antibody (Invitrogen) overnight at $4{ }^{\circ} \mathrm{C}$. The next day, after a 1-h wash with PBST, the sections were further incubated with secondary goat anti-mouse IgG antibody. For cell culture analysis, Hep3B cells were incubated with BrdU at a final concentration of $20 \mu \mathrm{M}$ for $2 \mathrm{~h}$. After a PBS wash, cells were fixed with $4 \%$ PFA for $5 \mathrm{~min}$, permeabilized with acetone:methanol (1:1) for $3 \mathrm{~min}$ on ice, then rehydrated in PBS for $5 \mathrm{~min}$. The following denaturing and antibody incubation steps were similar to those of the staining of the cryosections.

\subsection{Cell Culture}

Hep3B, Hek293, U2OS, and HeLa cells were cultured in 90\% Dulbecco's modified Eagle medium (DMEM; Gibco) supplemented with 10\% fetal bovine serum (FBS; Gibco, Grand Island, NY, USA), 
and maintained at $37{ }^{\circ} \mathrm{C}$ in a humidified incubator supplied with $5 \% \mathrm{CO}_{2}$. The hTERT-RPE- 1 cells were grown in DMEM/F12 (Gibco) media supplemented with 10\% FBS.

\subsection{RNA Interference and Immunofluorescence}

SiRNAs against human ANKRD45, were purchased from GenePharma (Shanghai, China). The four siRNA sequences were: siRNA1: 5'-GCCCAAGAACCAGAGGAAA-3', siRNA2: 5'-GGAUCCUGAGAAUCCUCAU-3', siRNA3: 5'-GGAAGAAAGGGCUCGAGAU-3' , and siRNA4: 5'-CCUUAAGGAAGACAAGAA-3' . A general sequence of 5'-UUCUCCGAACGUGUCACGU-3' was used as a negative control. All siRNAs were transfected at a final concentration of $50 \mu \mathrm{M}$ with Lipofectamine ${ }^{\mathrm{TM}}$ RNAiMAX (Invitrogen) following the manufacturer's protocol. The following antibodies were used for Western blot analysis: rabbit polyclonal antibodies against human ANKRD45 (Abcam, ab97878, Cambridge, MA, USA), cleaved PARP (Cell Signaling, Danvers, MA, USA), and actin (Beyotime Biotech Inc., Beijing, China).

For immunofluorescence, cells were fixed in 4\% PFA at RT for $10 \mathrm{~min}$, then treated with cold methanol at $-20^{\circ} \mathrm{C}$ for $5 \mathrm{~min}$. After two washes with PBS, the slides were permeabilized using $0.25 \%$ Triton-X-100 for $5 \mathrm{~min}$, blocked with 3\% BSA in PBS for $45 \mathrm{~min}$, then incubated with anti-ANKRD45 and anti- $\alpha$-tubulin antibodies in $3 \%$ BSA in PBS overnight at $4{ }^{\circ} \mathrm{C}$. After washing with PBS, the cells were incubated with secondary antibodies for $4 \mathrm{~h}$ at RT. All the images were captured using a Lecia Sp8 confocal microscope. We used two antibodies (Abcam, ab97878 and Invitrogen, PA5-21796) to detect ANKRD45 localization in cultured cells. Both of these two antibodies exhibited a similar staining pattern to that of ANKRD45 proteins. We only showed the immunofluorescence images in the paper with anti-ANKRD45 antibodies from Abcam (Cambridge, MA, USA).

\subsection{Knockdown of ANKRD45 with the CRISPR/Cas9 System}

Two sgRNAs that target the human ANKRD45 gene were designed with the following sequences: sgRNA-1: 5'-CTTAGAAGAAGACATCGTT-3' and sgRNA-2: 5'-TTGTAAAAGGGGGTTCTTA-3'. The target sequences were cloned into the sgRNA expression vector via BbsI restriction enzyme digestion. Each gRNA expression construct was transfected together with the pSpCas9 expression vector into Hep3B cells. The empty sgRNA expression vector was transfected together with pSpCas9 as a negative control. After $48 \mathrm{~h}$ of transfection, the expression level of ANKRD45 was examined by immunoblot analysis.

\subsection{Time-Lapse Assay}

HeLa cells were first transfected with control or ANKRD 45 siRNA for $48 \mathrm{~h}$ as described above. After that, cells were plated on $20 \mathrm{~mm}$ glass-bottomed dishes. Time-lapse images were recorded at $5 \mathrm{~min}$ intervals for $12 \mathrm{~h}$ using a Nikon A1 laser scanning confocal microscope equipped with $20 \times$ objective lens. The movies were generated using the Image J software (NIH Image, Bethesda, MD, USA).

\subsection{CCK8 Assay}

Cell proliferation was assessed using the CCK-8 assays kit (Sigma), which uses a water-soluble tetrazolium salt to quantify the number of live cells by producing an orange formazan dye upon bio-reduction in the presence of an electron carrier. Briefly, Hep3B cells were transfected with $50 \mu \mathrm{M}$ control or ANKRD45 siRNA and seeded onto 96-well plates at an initial density of $1 \times 10^{3}$ cells/well. At 1, 2,3, and 4 days, $10 \mu \mathrm{L}$ of CCK-8 solution was added to each well of the plate. After a one-hour incubation at $37^{\circ} \mathrm{C}$, spectrophotometric readings (A450 nm) were obtained on a microplate reader (Bio-Rad Laboratories, Hercules, CA, USA). Each experiment was repeated at least three times, and the data presented represent the mean of all measurements. 


\subsection{Cell Cycle and Annexin V Apoptosis Assay}

Cell cycle distributions were determined by flow cytometry through staining of the cells with propidium iodide (PI). Briefly, Hep3B cells were harvested at $48 \mathrm{~h}$ or $72 \mathrm{~h}$ after siRNA treatment, then washed with $1 \times \mathrm{PBS}$ and resuspended in $75 \%$ cold ethanol at $4{ }^{\circ} \mathrm{C}$ overnight. After a further wash with $1 \times$ PBS, fixed cells were treated with RNase for $30 \mathrm{~min}$, and incubated with $30 \mu \mathrm{g} / \mathrm{mL}$ PI for one hour in the dark. The cells were analyzed with a Beckman Coulter Cytomics FC500MPC Flow Cytometry Analyzer.

The percentage of apoptotic cells was assessed with an eBioscience ${ }^{\mathrm{TM}}$ Annexin V-FITC Apoptosis Detection Kit according to the manufacturer's instructions (Invitrogen, 88-8005-72). Hep3B cells transfected with siRNA or sgRNA constructs were harvested and washed with cold PBS, then resuspended in $100 \mu \mathrm{L}$ binding buffer containing $5 \mu \mathrm{L}$ Annexin V-FITC. After 15 min incubation in the dark, cells were centrifuged at $1000 \mathrm{rpm}$ for $5 \mathrm{~min}$ and resuspended in $500 \mu \mathrm{L}$ binding buffer containing $5 \mu \mathrm{L}$ of PI for $15 \mathrm{~min}$ in the dark at RT. The apoptosis was analyzed by a Beckman Coulter Cytomics FC500MPC Flow Cytometry Analyzer.

\section{Results}

\subsection{Expression of Ankrd45 Is Enriched in Ciliated Organs}

When searching for genes involved in ciliogenesis, we identified ankrd45 as a candidate gene using whole-mount in situ-hybridization gene expression screening. The staining of ankrd45 was first weakly detected in the dorsal forerunner cells at $80 \%$ epiboly to bud stages (Figure 1A). At the 10-somite stage, strong staining was detected in the otic placodes, Kupffer's vesicles, and floor plates (Figure 1B). Later, at $24 \mathrm{~h}$ post-fertilization (hpf), ankrd45 was mainly expressed in those organs rich in motile cilia, including otic vesicles, pronephric ducts, and floor plates (Figure 1C) [17]. At later stages, ankrd45 displayed a ubiquitous expression pattern. Real-time PCR results showed that the expression of ankrd45 persists into the adult stage with higher expression in the brain, liver, and testis (Figure 1D).

\subsection{Ankrd45 Is Dispensable for Zebrafish Development}

Zebrafish Ankrd45 contains 224 amino acids with two ankyrin repeats, which are conserved between humans and zebrafish (Figure 1E). The expression pattern suggests that Ankrd45 might play a role during ciliogenesis. To elucidate this, we generated zebrafish ankrd 45 mutants using the CRISPR/Cas9 system and recovered a mutant allele containing a 5 bp deletion in the target area (Figure 1E). This deletion introduced a new restriction site in the target region, and led to a premature termination of translation that encodes only the $\mathrm{N}$-terminal 90 amino acids of the wild-type protein (Figure S1A,B). Further in situ hybridization results suggested that mutant mRNA was degraded due to nonsense-mediated mRNA decay, a common phenomenon in zebrafish mutants (Figure S1C,D) [24,25].

Next, we compared cilia in several organs of mutant and wild-type larvae, including olfactory pits, pronephric ducts, spinal canals, otic vesicles, and neuromasts, by immunofluorescence with anti-glycylated tubulin antibodies. Surprisingly, cilia developed normally in these tissues, and the length of mutant cilia was comparable to those in wild type (Figure 1F,J). Furthermore, ankrd45 mutants were viable and fertile. These results suggest that Ankrd45 is dispensable for ciliogenesis, as well as zebrafish development.

\subsection{Liver Specific Expression of Kras ${ }^{G 12 V}$ Induced Larger Livers in Ankrd45 Mutants}

Considering that many ANK proteins play a role during cell cycle regulation, we investigated whether loss of function of Ankrd45 could lead to cell proliferation defects in zebrafish larvae. Both real-time PCR and fluorescence in situ hybridization results suggested that ankrd45 displayed a higher expression level in the liver (Figure 1D, Figure 2A-B"). We further generated ankrd45 mutants carrying a doxycycline-inducible transgenic line, Tg (fabp10:rtTA2s-M2; TRE2:EGFP-kras ${ }^{\text {G12V }}$ ), which 
contained a liver-specific double transgene to induce the expression of EGFP-Kras ${ }^{\mathrm{G} 12 \mathrm{~V}}$ specifically in the liver [21,22]. KRAS is one of the three RAS isoforms that is most frequently mutated in human cancers. The KRAS ${ }^{\mathrm{G} 12 \mathrm{~V}}$ mutation reduces the ability of the RAS small GTPase to hydrolyze to GDP, rendering it constitutively active for downstream signaling [22,26]. Upon induction, as reported previously, enlarged and hyperplastic livers developed in both wild-type and ankrd45 mutant larvae (Figure 2C,D). We compared the size of the liver between mutant and wild-type larvae from day 1 to day 5 after doxycycline treatment. Unexpectedly, ankrd45 mutants developed a larger liver than wild-type larvae after treatment (Figure 2C,D). The average liver size was almost double in ankrd45 mutants compared to that in control larvae (Figure 2C). We further injected wild-type ankrd45 mRNA into the mutant larvae and found that the formation of the enlarged liver was diminished in the mutants (Figure S2A). These data suggested that Ankrd45 protein is involved in the Kras ${ }^{\mathrm{G} 12 \mathrm{~V}_{\text {-induced }}}$ liver tumor growth.

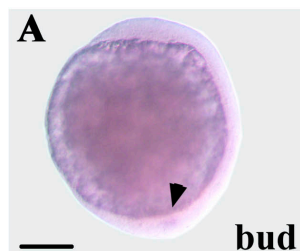

B

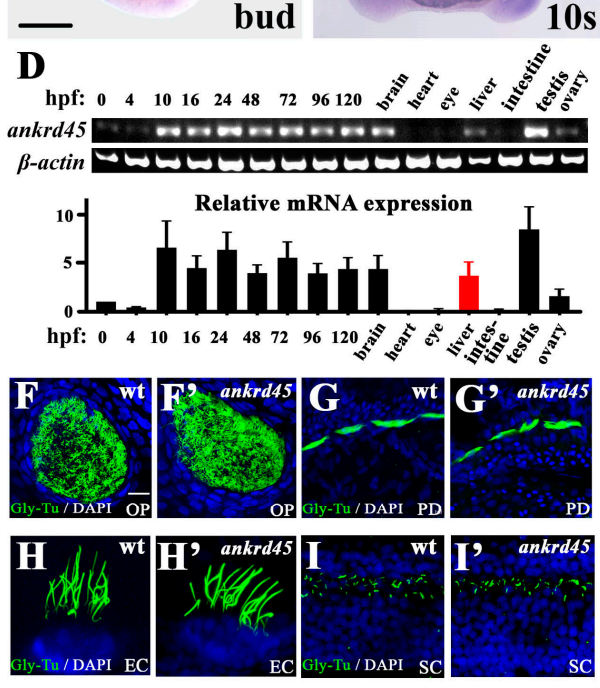

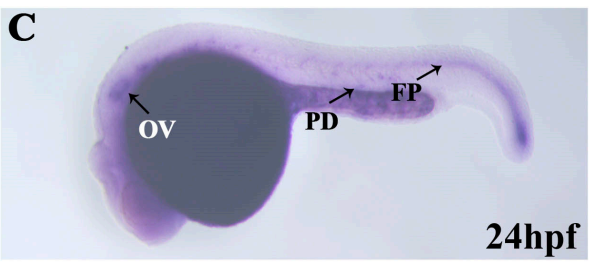

E
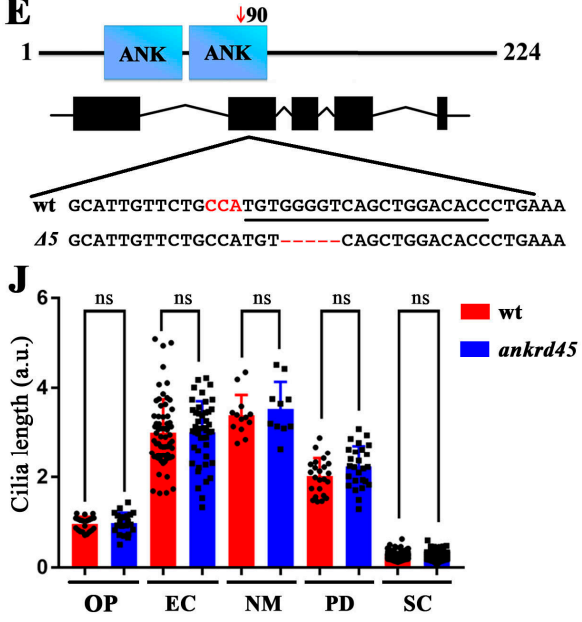

Figure 1. Expression pattern of ankrd45 and cilia development in ankrd45 mutants. (A-C) Whole-mount in situ hybridization results showing the expression of ankrd45 at different stages. (A) At the bud stage, ankrd45 is expressed in the dorsal forerunner cells (arrowhead). (B) At the 10-somite stage, ankrd45 is expressed in the otic vesicle (OV), Kupffer's vesicle (KV), and floor plate (FP). (C) ankrd45 is enriched in the otic vesicle, the pronephric duct (PD), and the floor plate at $24 \mathrm{hpf}$. (D) Real-time PCR showing the expression of ankrd45 at different stages or adult tissues as indicated. Bottom: bar graph showing the relative expression levels of ankrd45 at different stages or adult tissues as indicated. (E) Diagram showing the genomic structure of the zebrafish ankrd45 gene. Bottom shows the sequences of wild-type and mutant alleles $(\Delta 5)$. Underlined sequence indicates the spCas 9 target region, and the PAM sequence is indicated in red. $\left(\mathbf{F}-\mathbf{I}^{\prime}\right)$ Confocal images showing the cilia in the olfactory pit $\left(\mathbf{F}, \mathbf{F}^{\prime}\right)$, pronephric $\operatorname{duct}\left(\mathbf{G}, \mathbf{G}^{\prime}\right)$, ear cristae $\left(\mathbf{H}, \mathbf{H}^{\prime}\right)$, and spinal cord $\left(\mathbf{I}, \mathbf{I}^{\prime}\right)$ in wild-type or mutant larvae visualized with anti-glycylated tubulin antibody. (J) Bar graph showing the length of cilia per arbitrary unit (a.u.) in different organs as indicated. OP, olfactory pit; EC, ear cristae; SC, spinal cord; NM, neuromast. Scale bars: $200 \mu \mathrm{m}$ in $\mathrm{A}-\mathrm{C} ; 20 \mu \mathrm{m}$ in $\mathrm{F}-\mathrm{I}^{\prime}$. ns, not significant.

\subsection{Enlarged Cystic Liver Is Due to Cell Degeneration in Kras ${ }^{G 12 V}$-Induced Ankrd45 Mutant}

To better understand the mechanisms underlying the enlarged liver development in the mutant, we further investigated the liver morphology using hematoxylin and eosin (H\&E) staining and oil red 
O staining on the histological sections. The oil red $\mathrm{O}$ staining showed no difference in lipid formation between mutant and wild-type embryos (data not shown). In wild-type embryos, H\&E staining results showed a hyperplastic liver due to Kras ${ }^{\mathrm{G} 12 \mathrm{~V}}$-induced cell proliferation. In contrast, multiple cyst-like areas were present throughout the mutant liver (Figure 2E,F). To further explain the reason for cyst formation, we compared cell apoptosis between mutant and wild-type larvae at different time points after induction. TUNEL assay results showed that the number of apoptotic cells increased significantly $(p<0.001)$ in the mutant liver at 3, 4, and 5 days post treatment (dpt) (Figure 2G-I, Figure S2B,D). In contrast, BrdU labeling results showed that the number of proliferating cells decreased substantially in the mutant (Figure 2J-L, Figure S2C,E). Noticeably, we observed significant loss of proliferating cells at five-days post treatment, compared with those at 3 and $4 \mathrm{dpt}$ (Figure S2E). These data suggest that loss of ankrd45 results in a large number of apoptotic cells at the expense of proliferating cells in the $\mathrm{Kras}^{\mathrm{G} 12 \mathrm{~V}}$-induced liver tumor, which leads to the formation of large areas of cystic degeneration and represents the main reason for enlarged livers in the mutants.
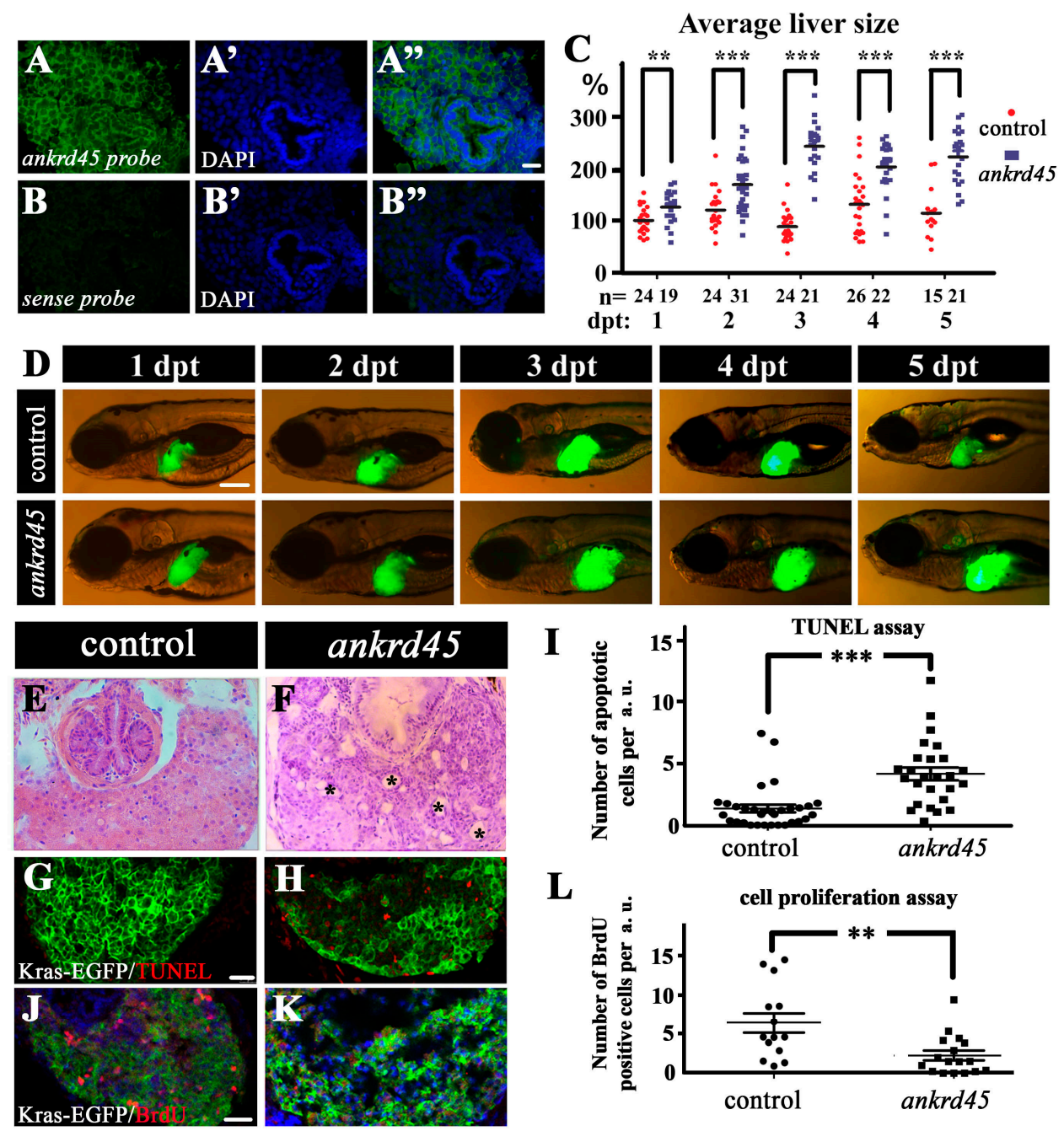

Figure 2. Liver-specific induction of $\mathrm{Kras}^{\mathrm{G} 12 V}$ results in larger livers in ankrd45 mutants. (A-B") Fluorescent in situ hybridization results showing the expression of ankrd45 in the liver of $5 \mathrm{dpf}$ wild-type zebrafish larvae. Sense probe of ankrd45 was used as a negative control (B-B"). (C) Statistical results showing the average size of livers in control larvae (EGFP-kras ${ }^{G 12 V}$ heterozygote transgene) or ankrd45 larvae (ankrd45 homozygous mutants carrying EGFP-kras ${ }^{G 12 V}$ heterozygote transgene) at different time points after doxycycline treatment as indicated. Each dot represents the size of one larva. The average size of the livers at 1 day post treatment (dpt) in the control larvae was set as $100 \%$. The 
number of larvae examined is shown at the bottom. (D) Representative images showing EGFP-Kras ${ }^{\mathrm{G} 12 \mathrm{~V}}$ expression in the livers of control and ankrd45 mutants at different time points after doxycycline treatment. (E,F) Hematoxylin and eosin staining results showing the multiple cyst-like regions (asterisks) in the livers of ankrd45 mutants after treatment. $(\mathbf{G}, \mathbf{H})$ Confocal images showing the apoptotic cells stained by the TUNEL assay. (I) Dotted graph showing the number of apoptotic cells in the livers of control and ankrd45 mutants 3 days after treatment. $(\mathbf{J}, \mathbf{K})$ Confocal images showing BrdU positive cells in the livers of control and mutant larvae as indicated. (L) Dotted graph showing the number of BrdU positive cells in the livers of control and ankrd45 mutants 3 days after treatment. Scale bars: $25 \mu \mathrm{m}$ in panels A-B", $200 \mu \mathrm{m}$ in panel D, and $25 \mu \mathrm{m}$ in panels G-K. ${ }^{* *} p<0.01,{ }^{* * *} p<0.001$.

\subsection{Dynamic Subcellular Localization of ANKRD45 During Mitosis}

Considering that high levels of Kras ${ }^{\mathrm{G} 12 \mathrm{~V}}$ expression can lead to hepatocellular carcinoma (HCC) in zebrafish and that KRAS ${ }^{G 12 V}$ is also the most popular mutation driving cancer cell proliferation $[19,22]$, we further analyzed the role of ANKRD45 using several human cancer cells. First, we analyzed subcellular localization of ANKRD45 using human hepatoma Hep3B cells. Interestingly, the subcellular localization of ANKRD45 was cell-cycle dependent. Although we did not detect any signals during interphase, ANKRD45 localized diffusely in the entire cytoplasm during metaphase (Figure 3A-A"). During anaphase and telophase, ANKRD45 was detected to be highly concentrated at the cleavage furrow region (Figure 3B-C"). ANKRD45 appeared to accumulate rapidly during cleavage-furrow formation and was enriched at the midbody ring at the later cytokinesis (Figure 3D,D"). Of note, ANKRD45 was distributed in a dotted manner at early stages of cell division and was later enriched in the apical region beneath the cell membrane, similar to those proteins that are essential for midbody ring formation $[27,28]$. We observed a similar localization pattern of ANKRD45 in several other cell lines, suggesting the conserved role of this protein during cell division (Figure S3A-D). Furthermore, we pre-incubated the antibody with recombinant human ANKRD45 peptide before antibody staining, such treatment completely blocked the fluorescence signal in the midbody (Figure S3E,F).

\subsection{Loss of ANKRD45 Results in Cell Proliferation Defects in Cultured Cells}

To further examine the role of ANKRD45 during cell division, we designed four ANKRD45 siRNAs to silence ANKRD45's expression. siRNA\#2 showed more than 50\% knockdown efficiency compared to other siRNAs in both Hep3B cells and HeLa cells (Figure 3E, Figure S3G,H). When treated with this siRNA, the fluorescence signal of ANKRD45 in the midbody ring decreased significantly $(p<0.001)$ (Figure 3F-H), which further confirmed the localization of ANKRD45.

Surprisingly, we observed robust, round-shaped, detached cells floating in the medium in siRNA-treated cells, indicating that these were apoptotic cells. To better describe this observation, we performed time-lapse microscopy to compare cell division between control and ANKRD45 siRNA-treated HeLa cells. In control siRNA-treated cells, cell cycle progressed through mitosis and cytokinesis normally within 2-3 h (Figure 4A, Supplementary Movie S1). In contrast, silencing of ANKRD45 resulted in cell division defects and cell death characterized by rounding up of the cell body and eventual detachment from the plate (Figure 4B, Supplementary Movie S2). Consistent with this, the cleaved form of PARP-1, a hallmark of cell apoptosis, was also increased in both HeLa and Hep3B cells (Figure 3E, Figure S3G). We stained these cells with Annexin V and performed flow cytometry analysis, which further confirmed cell apoptosis after siRNA treatment (Figure 4C,D). 




Figure 3. Subcellular localization of ANKRD45 during mitosis. (A-D") Subcellular localization of ANKRD45 during mitosis in human hepatoma Hep3B cells. The distribution of ANKRD45 was visualized with anti-ANKRD45 antibody (green). The cytoskeleton was stained by anti- $\alpha$-tubulin antibody (red) and nuclei were labeled with DAPI (blue). (E) Hep3B cells were treated with control or four siRNAs against ANKRD45 and analyzed with Western blotting using ANKRD45 antibody or a cleaved form of the PARP antibody. The $\alpha$-tubulin antibody was used as a loading control. Bottom bar graph shows the statistical results of ANKRD45 expression in control or siRNA-treated cells from the immunoblots results. $(\mathbf{F}, \mathbf{G})$ Confocal images showing the localization of ANKRD45 in the midbody during cytokinesis in control (F) or siRNA\#2 (G) treated HeLa cells. (H) Quantitative analysis of relative ANKRD45 expression in the midbody ring of control or siRNA\#2-treated HeLa cells. NC, negative control. Scale bars: $5 \mu \mathrm{m}$. ${ }^{* * *} p<0.001$. 
Next, we performed a cell proliferation assay using a cell counting kit-8 (CCK-8) to evaluate cell viability in ANKRD45 siRNA-treated cells. Compared with the control group, knockdown of ANKRD45 expression dramatically affected cell viability (Figure 4E). Flow cytometry analysis results suggested that the percentages of cells in G0/G1 stages were greatly increased at both $48 \mathrm{~h}$ and $72 \mathrm{~h}$ after treatment, suggesting that knockdown of ANKRD45 expression delayed cell cycle progression (Figure 4F,G). Finally, BrdU incorporation and TUNEL assay further suggested that cell proliferation was diminished and cell apoptosis was induced after siRNA treatment (Figure $4 \mathrm{H}-\mathrm{J}$ ).

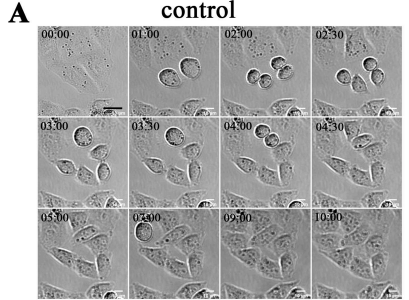

B

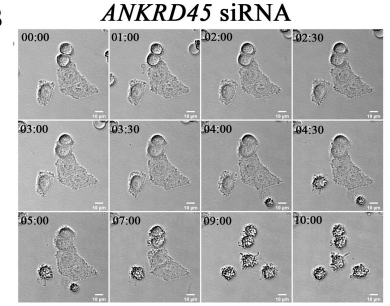

C
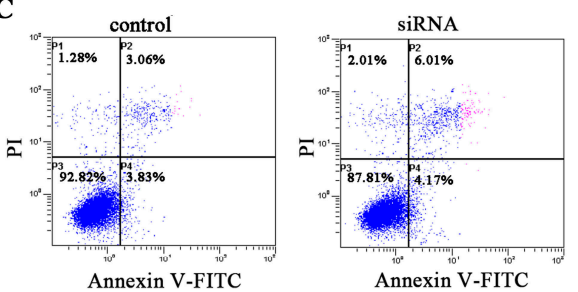

D

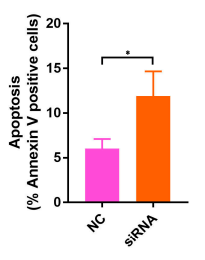

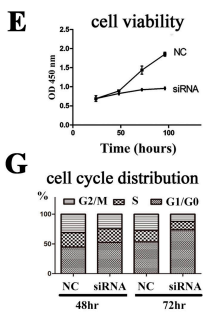

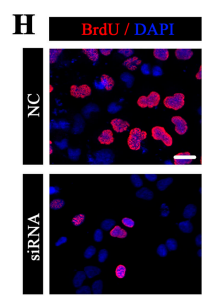

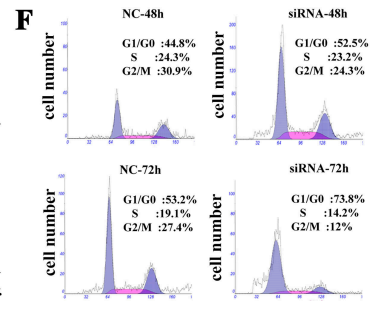

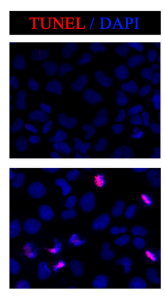

Figure 4. Knockdown of ANKRD45 results in cell cycle defects. (A,B) DIC (Differential Interference Contrast) still images obtained from time-lapse microscopy showing HeLa cells undergoing cell division. Compared with control siRNA-treated cells (A), knockdown of ANKRD45 (B) resulted in the delay of cell division and cell death. (C,D) Flow cytometry analysis of Annexin V-FITC and propidium iodide staining of the control and ANKRD45 siRNA-treated Hep3B cells. The percentage of apoptotic cells increased significantly after siRNA treatment (D). (E) Cell proliferation of Hep3B cells analyzed by CCK-8 assay. (F,G) Flow cytometry cell cycle analysis of Hep3B cells treated with control or siRNA. The percentage of cells in different phases of the cell cycle are shown in $(G)$. (H) Confocal images showing proliferating and apoptotic Hep3B cells visualized by BrdU incorporation and the TUNEL assay. (I) Quantitative results of the percentage of BrdU positive cells in control or siRNA-treated Hep3B cells. (J) Quantitative results of the percentage of apoptotic cells from the TUNEL assay. NC, negative control. Scale bars: $25 \mu \mathrm{m} .{ }^{*} p<0.05,{ }^{* *} p<0.01,{ }^{* * *} p<0.001$.

Considering that siRNA-mediated knockdown may cause off-target effects, we further designed two single-guide RNAs (sgRNAs) to transiently knockdown ANKRD45 expression using the CRISPR/Cas9 system (Figure 5A). The efficiency of these two sgRNAs was confirmed by T7EI assay (Figure 5B). Western blot with ANKRD45 antibody showed that the expression of ANKRD45 was successfully knocked down in both sgRNA transfected cells (Figure 5C). Again, we observed a large number of dead cells at $72 \mathrm{~h}$ after sgRNA transfection (Figure 5D-F). We stained the transfected cells with Annexin V, which further confirmed the increase of apoptotic cells (Figure 5G,H). Finally, the expression level of the cleaved form of PARP-1 was also upregulated when ANKRD45 was knocked down (Figure 5C).

Together, both the siRNA- and sgRNA-mediated knockdown results suggest that ANKRD45 plays an important role during cell division, and ANKRD45 deficiency can lead to cell proliferation defects and finally result in cell apoptosis, similar to results seen in zebrafish ankrd45 mutants. 
A

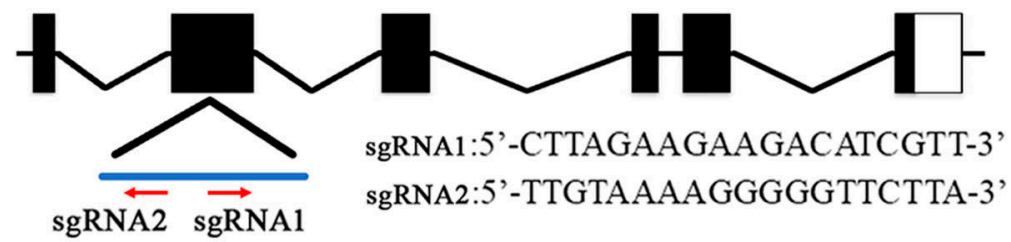

B
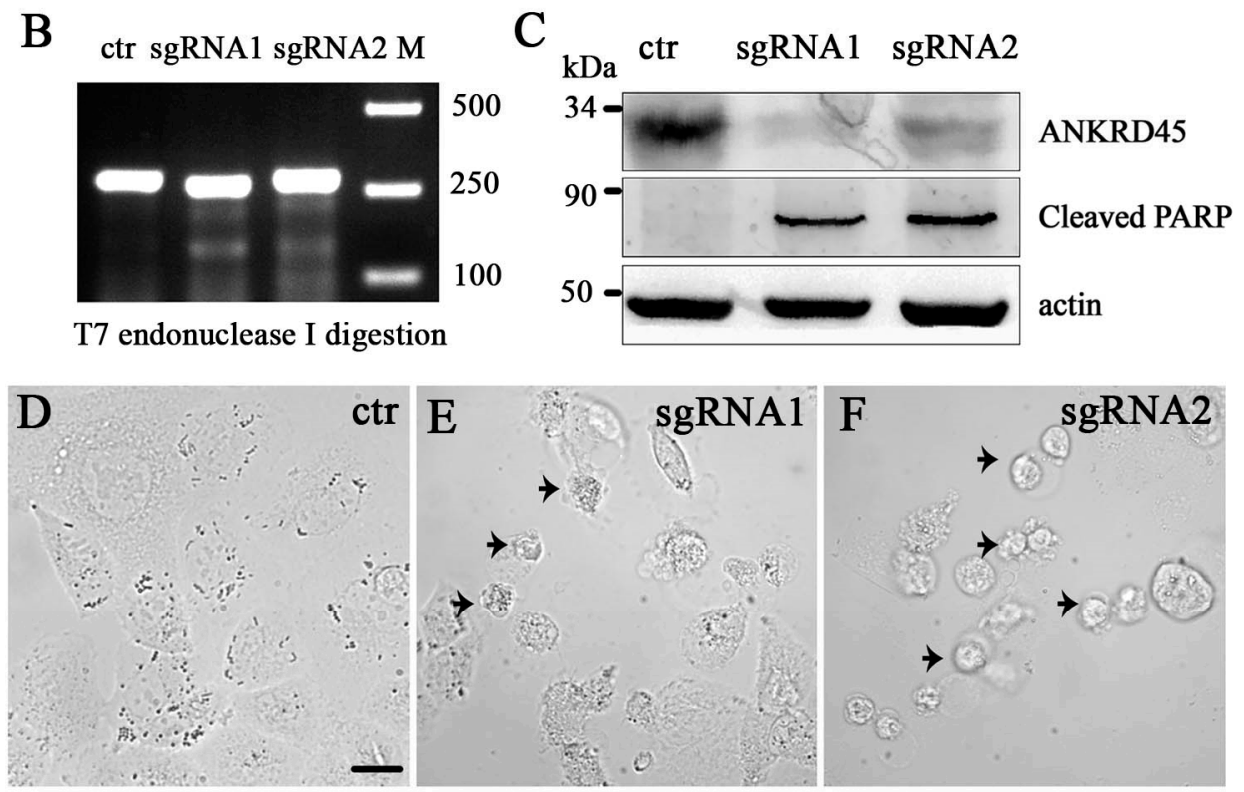

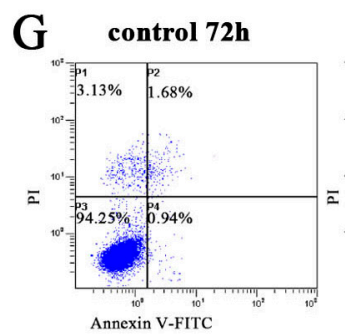

control 96h

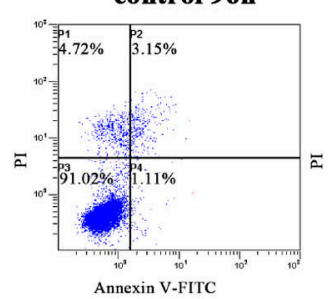

Annexin V-FITC
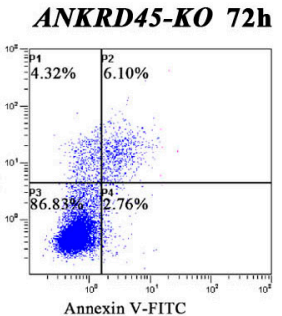

ANKRD45-KO 96h

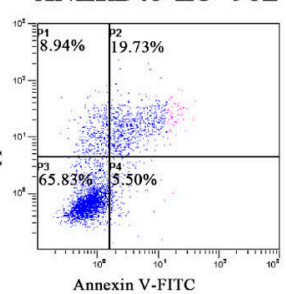

$\mathrm{H}$

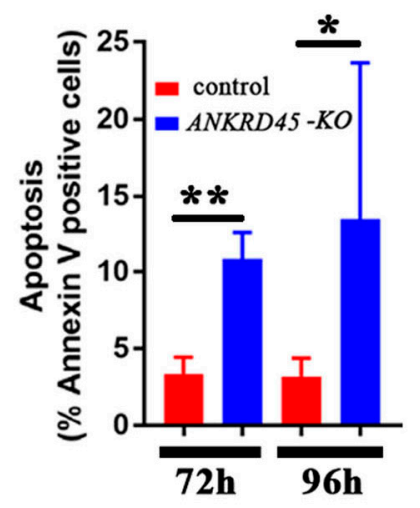

Figure 5. Transient knockdown of ANKRD45 with the CRISPR/Cas9 system results in cell apoptosis. (A) Genomic structure of the human ANKRD45 gene and sequences of two sgRNAs. Red arrows indicate the position and direction of the two sgRNAs. (B) Efficiency of the two sgRNAs analyzed by T7 endonuclease I digestion (T7EI). (C) Western blotting results showing the knockdown efficiency of two ANKRD45-targeting sequences. Both targets led to the reduction of ANKRD45 expression and increased the level of the cleaved PARP. (D-F) DIC images showing Hep3B cells transfected with control or ANKRD45 targeting constructs containing different sgRNAs as indicated. Arrowheads represent the apoptotic cells. (G) Flow cytometry analysis of Annexin V-FITC and propidium iodide staining of Hep3B cells transfected with sgRNA or empty vectors. (H) Bar graph showing the percentages of Annexin $\mathrm{V}$ positive cells at $72 \mathrm{~h}$ and $96 \mathrm{~h}$ after transfection with control or sgRNAs as indicated. ${ }^{*} p<0.05,{ }^{* *} p<0.01$, Scale bar: $25 \mu \mathrm{m}$. 


\section{Discussion}

Ankyrin repeat-containing proteins are one of the most common protein families present in eukaryotic cells and they play a role in various biological functions. Here, we reported the function of a novel ANK protein, ANKRD45, which contains two ankyrin repeats. The expression pattern of ankrd45 in early zebrafish embryos was tissue specific with high enrichment in ciliated organs, suggesting that Ankrd45 possibly plays a role during cilia development. Indeed, ANKRD45 was also identified recently from proteomics analysis by comparison of ciliary proteins in different species [29]. Unexpectedly, homozygous ankrd45 mutants were viable and showed no apparent defects. All the cilia detected were grossly normal in ankrd45 mutants. Although we cannot rule out subtle defects, our results suggest that Ankrd45 is not essential for ciliogenesis in zebrafish. Interestingly, ankrd45 mutants displayed abnormal reaction during stress response, such as $\mathrm{Kras}^{\mathrm{G} 12 \mathrm{~V}}$-induced tumor formation. Overexpression of the oncogenic Kras ${ }^{\mathrm{G} 12 \mathrm{~V}}$ could promote tumor formation when strongly expressed in the liver in wild-type zebrafish [22]. The induction of enlarged livers seems to be more prominent in ankrd45 mutant larvae. However, our histological results suggest that the enlarged livers were indeed due to the presence of a large amount of cystic degeneration due to cell apoptosis. These data suggest that Ankrd45 participates in the regulation of Kras ${ }^{\mathrm{G} 12 \mathrm{~V}}$-induced cell proliferation. Similarly, ANKRD45 is also important for cell proliferation in several cancer cells, including human hepatoma Hep3B cells, which are relatively similar to the Kras ${ }^{\mathrm{G} 12 \mathrm{~V}}$-induced liver cells.

Although deficiency of Ankrd45 proteins resulted in cell division defects both in $\mathrm{Kras}^{\mathrm{G} 12 \mathrm{~V}}$-induced liver cells and cultured cancer cells, we did not observe any cell proliferation defects in ankrd45 mutants. One possible explanation is that the embryos are complex, multicellular organisms with elaborate gene regulation systems, which can trigger multiple signaling pathways to compensate for the loss of Ankrd45 in the mutant larvae [30]. Under stress conditions, such as rapid cell division in tumor cells, which might require high ANKRD45 levels, the gene regulation systems fail to fully compensate for the loss of ANKRD45, which eventually leads to cell apoptosis. The high requirement of ANKRD45 during cancer cell proliferation may be worthy of further attention to evaluate the potential target effects during therapeutic trials.

The dynamic subcellular localization of ANKRD45 during cell cycle progression is consistent with its role in cell proliferation. Time-lapse microscopy showed that cell division was arrested during the cell cycle, resulting in cell death at interphase and/or metaphase, which suggested that ANKRD45 is required for cell division both in early and late mitotic stages. Currently, the mechanism by which ANKRD45 regulates mitosis is still unclear. ANKRD45 was slightly enriched beneath the plasmid membrane during the cleavage furrow formation and later in the midbody ring (Figure 3A-D"). Such a distribution was similar to that of some actin binding proteins, such as ezrin/radixin/moesin (ERM) proteins, which are involved in the regulation of membrane morphology [28,31]. Future studies will be necessary to decipher the mechanisms by which ANKRD45 regulates mitosis.

Supplementary Materials: The following are available online at http://www.mdpi.com/2073-4425/10/6/462/s1, Figure S1: Identification of ankrd45 mutants, Figure S2: Liver-specific induction of Kras ${ }^{\mathrm{G} 12 \mathrm{~V}}$ in ankrd45 mutants, Figure S3: Localization of ANKRD45 in different cell lines, Movie S1: Time-lapse microscopy of HeLa cells treated with control siRNA, Movie S2: Time-lapse microscopy of HeLa cells treated with ANKRD45 siRNA.

Author Contributions: Conceptualization, Y.K. and C.Z.; formal analysis, Y.K., H.X., and C.Z.; investigation, Y.K. and H.X.; data curation, Y.K. and C.Z.; writing-original draft preparation, Y.K. and H.X.; writing-review and editing, C.Z.; funding acquisition, C.Z.

Funding: This work was supported by the Marine S\&T Fund of Shandong Province for the Pilot National Laboratory for Marine Science and Technology (Qingdao) (No. 2018SDKJ0406-2) and by grants from the Fundamental Research Funds for Central Universities (Grants 201941004) and the National Natural Science Foundation of China (Nos. 31772456).

Acknowledgments: We thank Zhiyuan Gong for providing the EGFP-kras ${ }^{G 12 V}$ transgenic line and Jinghao Sheng and Long Zhang for providing constructs for cell analysis. We also thank members of the Zhao lab for the helpful comments on an earlier version of this manuscript.

Conflicts of Interest: The authors declare no conflict of interest. 


\section{References}

1. Barrick, D.; Ferreiro, D.U.; Komives, E.A. Folding landscapes of ankyrin repeat proteins: Experiments meet theory. Curr. Opin. Struct. Biol. 2008, 18, 27-34. [CrossRef] [PubMed]

2. Mosavi, L.K.; Cammett, T.J.; Desrosiers, D.C.; Peng, Z.Y. The ankyrin repeat as molecular architecture for protein recognition. Protein Sci. Publ. Protein Soc. 2004, 13, 1435-1448. [CrossRef] [PubMed]

3. Breeden, L.; Nasmyth, K. Similarity between cell-cycle genes of budding yeast and fission yeast and the notch gene of drosophila. Nature 1987, 329, 651-654. [CrossRef] [PubMed]

4. Lux, S.E.; John, K.M.; Bennett, V. Analysis of cdna for human erythrocyte ankyrin indicates a repeated structure with homology to tissue-differentiation and cell-cycle control proteins. Nature 1990, 344, 36-42. [CrossRef] [PubMed]

5. Kojic, S.; Radojkovic, D.; Faulkner, G. Muscle ankyrin repeat proteins: Their role in striated muscle function in health and disease. Crit. Rev. Clin. Lab. Sci. 2011, 48, 269-294. [CrossRef] [PubMed]

6. Russo, A.A.; Tong, L.; Lee, J.O.; Jeffrey, P.D.; Pavletich, N.P. Structural basis for inhibition of the cyclin-dependent kinase cdk6 by the tumour suppressor p16ink4a. Nature 1998, 395, 237-243. [CrossRef] [PubMed]

7. Ortega, S.; Malumbres, M.; Barbacid, M. Cyclin d-dependent kinases, ink4 inhibitors and cancer. Biochim. Biophys. Acta 2002, 1602, 73-87. [CrossRef]

8. Joutel, A.; Corpechot, C.; Ducros, A.; Vahedi, K.; Chabriat, H.; Mouton, P.; Alamowitch, S.; Domenga, V.; Cecillion, M.; Marechal, E.; et al. Notch3 mutations in cadasil, a hereditary adult-onset condition causing stroke and dementia. Nature 1996, 383, 707-710. [CrossRef]

9. Braune, E.B.; Lendahl, U. Notch-A goldilocks signaling pathway in disease and cancer therapy. Discov. Med. 2016, 21, 189-196.

10. Baker, R.G.; Hayden, M.S.; Ghosh, S. NF-kB, inflammation, and metabolic disease. Cell Metab. 2011, 13, 11-22. [CrossRef]

11. Kondylis, V.; Kumari, S.; Vlantis, K.; Pasparakis, M. The interplay of IKK, NF-kB and RIPK1 signaling in the regulation of cell death, tissue homeostasis and inflammation. Immunol. Rev. 2017, 277, 113-127. [CrossRef] [PubMed]

12. Perillo, B.; Di Santi, A.; Cernera, G.; Ombra, M.N.; Castoria, G.; Migliaccio, A. Nuclear receptor-induced transcription is driven by spatially and timely restricted waves of ROS. The role of Akt, IKK $\alpha$, and DNA damage repair enzymes. Nucleus 2014, 5, 482-491. [CrossRef] [PubMed]

13. Perillo, B.; Di Santi, A.; Cernera, G.; Ombra, M.N.; Castoria, G.; Migliaccio, A. Phosphorylation of H3 serine 10 by IKK $\alpha$ governs cyclical production of ROS in estrogen-induced transcription and ensures DNA wholeness. Cell Death Differ. 2014, 21, 1503. [CrossRef] [PubMed]

14. Otto, E.A.; Schermer, B.; Obara, T.; O’Toole, J.F.; Hiller, K.S.; Mueller, A.M.; Ruf, R.G.; Hoefele, J.; Beekmann, F.; Landau, D.; et al. Mutations in invs encoding inversin cause nephronophthisis type 2, linking renal cystic disease to the function of primary cilia and left-right axis determination. Nat. Genet. 2003, 34, 413-420. [CrossRef] [PubMed]

15. Singla, V.; Reiter, J.F. The primary cilium as the cell's antenna: Signaling at a sensory organelle. Science 2006, 313, 629-633. [CrossRef] [PubMed]

16. Reiter, J.F.; Leroux, M.R. Genes and molecular pathways underpinning ciliopathies. Nat. Rev. Mol. Cell Biol. 2017, 18, 533-547. [CrossRef] [PubMed]

17. Song, Z.; Zhang, X.; Jia, S.; Yelick, P.C.; Zhao, C. Zebrafish as a model for human ciliopathies. J. Genet. Genom. = Yi Chuan Xue Bao 2016, 43, 107-120. [CrossRef]

18. Zhang, X.; Jia, S.; Chen, Z.; Chong, Y.L.; Xie, H.; Feng, D.; Wu, X.; Song, D.Z.; Roy, S.; Zhao, C. Cilia-driven cerebrospinal fluid flow directs expression of urotensin neuropeptides to straighten the vertebrate body axis. Nat. Genet. 2018, 50, 1666-1673. [CrossRef]

19. Li, S.; Balmain, A.; Counter, C.M. A model for ras mutation patterns in cancers: Finding the sweet spot. Nat. Rev. Cancer 2018, 18,767-777. [CrossRef]

20. Pylayeva-Gupta, Y.; Grabocka, E.; Bar-Sagi, D. Ras oncogenes: Weaving a tumorigenic web. Nat. Rev. Cancer 2011, 11, 761-774. [CrossRef] 
21. Chew, T.W.; Liu, X.J.; Liu, L.; Spitsbergen, J.M.; Gong, Z.; Low, B.C. Crosstalk of Ras and Rho: Activation of rhoa abates Kras-induced liver tumorigenesis in transgenic zebrafish models. Oncogene 2014, 33, 2717-2727. [CrossRef] [PubMed]

22. Nguyen, A.T.; Emelyanov, A.; Koh, C.H.; Spitsbergen, J.M.; Lam, S.H.; Mathavan, S.; Parinov, S.; Gong, Z. A high level of liver-specific expression of oncogenic Kras(V12) drives robust liver tumorigenesis in transgenic zebrafish. Dis. Models Mech. 2011, 4, 801-813. [CrossRef] [PubMed]

23. Feng, D.; Chen, Z.; Yang, K.; Miao, S.; Xu, B.; Kang, Y.; Xie, H.; Zhao, C. The cytoplasmic tail of rhodopsin triggers rapid rod degeneration in kinesin-2 mutants. J. Biol. Chem. 2017, 292, 17375-17386. [CrossRef] [PubMed]

24. El-Brolosy, M.A.; Kontarakis, Z.; Rossi, A.; Kuenne, C.; Gunther, S.; Fukuda, N.; Kikhi, K.; Boezio, G.L.M.; Takacs, C.M.; Lai, S.L.; et al. Genetic compensation triggered by mutant mrna degradation. Nature 2019, 568, 193-197. [CrossRef] [PubMed]

25. Ma, Z.; Zhu, P.; Shi, H.; Guo, L.; Zhang, Q.; Chen, Y.; Chen, S.; Zhang, Z.; Peng, J.; Chen, J. PTC-bearing mrna elicits a genetic compensation response via Upf3a and COMPASS components. Nature 2019, 568, $259-263$. [CrossRef] [PubMed]

26. Karreth, F.A.; Tuveson, D.A. Modelling oncogenic ras/raf signalling in the mouse. Curr. Opin. Genet. Dev. 2009, 19, 4-11. [CrossRef] [PubMed]

27. Pohl, C.; Jentsch, S. Final stages of cytokinesis and midbody ring formation are controlled by bruce. Cell 2008, 132, 832-845. [CrossRef]

28. Sato, N.; Yonemura, S.; Obinata, T.; Tsukita, S.; Tsukita, S. Radixin, a barbed end-capping actin-modulating protein, is concentrated at the cleavage furrow during cytokinesis. J. Cell Biol. 1991, 113, 321-330. [CrossRef]

29. Sigg, M.A.; Menchen, T.; Lee, C.; Johnson, J.; Jungnickel, M.K.; Choksi, S.P.; Garcia, G., 3rd; Busengdal, H.; Dougherty, G.W.; Pennekamp, P.; et al. Evolutionary proteomics uncovers ancient associations of cilia with signaling pathways. Dev. Cell 2017, 43, 744-762. [CrossRef]

30. Rossi, A.; Kontarakis, Z.; Gerri, C.; Nolte, H.; Holper, S.; Kruger, M.; Stainier, D.Y. Genetic compensation induced by deleterious mutations but not gene knockdowns. Nature 2015, 524, 230-233. [CrossRef]

31. Pelaseyed, T.; Bretscher, A. Regulation of actin-based apical structures on epithelial cells. J. Cell Sci. 2018, 131, jcs221853. [CrossRef] [PubMed]

(C) 2019 by the authors. Licensee MDPI, Basel, Switzerland. This article is an open access article distributed under the terms and conditions of the Creative Commons Attribution (CC BY) license (http://creativecommons.org/licenses/by/4.0/). 